\section{ORAL MEDICINE}

\section{Zinc gluconate in the treatment of} dysgeusia - a randomized clinical trial

Heckmann SM, Hujoel P et al J Dent Res 2005; 84: 35-38

Zinc may improve the experience and psychological mood of patients with altered gustatory sensation.

It is not clear whether zinc can improve dysgeusia. Previous studies of dysgeusia have suffered from small sample size and inclusion of differing causes of the condition. In this study, 50 patients with a diagnosis of idiopathic dysgeusia were randomized to zinc supplementation or placebo for 3 months. Another 66 dysgeusic patients not included in the study over the same recruitment period had various concomitant conditions such as dental material allergies and burning mouth syndrome.

There were significant differences between groups after treatment, both in terms of identifying the 4 basic tastes of sweet, sour, salty and bitter, and in self-assessment of gustatory function. The zinc supplementation group also improved significantly in depression scores. Serum zinc levels did not differ, perhaps because the element is rapidly transferred into cells. There was, however, an increase in salivary calcium level, perhaps because of an effect of zinc absorption.

doi:10.1038/sj.bdj.4812346.

CONSERVATIVE DENTAL SURGERY

The timing of subsequent treatment for teeth restored with large amalgams and crowns: factors related to the need for subsequent treatment

Kolker JL, Damiano PC et al. J Dent Res 2004; 83: 854-858

Crown survival was longer than that of large amalgam restorations, but the latter could be enhanced by other factors.

Teeth which have lost considerable coronal tissue may be treated with large amalgam restorations, in some cases followed by crowns. This study retrospectively compared survival rates of such restorations with and without crowns over a 10 year period in 737 teeth treated without root canal therapy in 1987-1988 in a US university. Survival of teeth receiving no subsequent treatment was compared with that of teeth subsequently receiving either root canal treatment or extraction.

With no further treatment, large amalgams had a 5-year survival rate of 0.52 , compared with 0.88 for crowns. Respective rates at 10 years were 0.46 and 0.77 . In teeth which were neither extracted nor root filled, respective 5 and 10 year survival rates for amalgams were 0.87 and 0.78 , and for crowns, 0.95 and 0.87. All differences were highly significant. The authors consider their findings should be viewed with caution, because not all relevant information was available.

ORAL SURGERY

\section{Patients referred to a specialist clinic because of suspected temporomandibular disorders: a survey of 3194 patients in respect of diagnoses, treatments, and treatment outcome}

Anastassaki A, Magnusson T Acta Odontol Scand 2004; 62: 183-192

Most patients responded quickly with conservative treatment methods.

While most patients with temporomandibular disorders (TMD) respond to some treatment, a variety of different treatments may be effective. Over an 8 yr period, 3194 patients (3/4 female) referred to a Swedish clinic were examined. Those with rare diagnoses, or without one according to ICD-9, were excluded (473), and in the remaining 2721, 4464 diagnoses were recorded.

Disc displacement was recorded in 29\%, tension headache in 19\%, myo-arthralgia in 18\%, arthritis in 14\%, myalgia in 11\%, osteoarthrosis in 10\%, nonspecific orofacial pain in 6\% and rheumatoid arthritis in 4\%. Therapeutic jaw exercises were prescribed for 75\%, and interocclusal appliances for 56\%. Women had greater severity of symptoms, and responded better to treatment than men. Regression analysis gave significant odds ratios for favourable outcome with 3 diagnoses: disc displacement without reduction, arthritis and myalgia; non-specific orofacial pain gave a poorer outcome.

doi:10.1038/sj.bdj.4812348

ENDODONTICS; HAEMATOLOGY

Detection of bacteraemias during nonsurgical root canal treatment

Savarrio L, Mackenzie D et al. J Dent 2005; 33: 293-303

Up to $30 \%$ of non-surgical root canal treatment may produce bacteraemia.

Previous research has suggested that some root canal instrumentation produces bacteraemia. In this study, aseptic techniques were used to access root canals in 30 subjects, and a sterile paper point was used to sample for microbes. Blood samples were taken before treatment, $5 \mathrm{~min}$ after access to canals, and $5 \mathrm{~min}$ after completion of the treatment.

In 23 root canal samples, a positive culture was obtained, and 32 types of organism were identified. There were negative blood cultures in 19 subjects at all 3 sampling times. Bacteraemia was present in 9 patients who did not have preoperative positive cultures. In 7 cases, the same species was found in root canals and blood cultures, and in 2 cases, genetic homogeneity was established for root canal and blood cultures. PCR identified 12 positive blood samples, 7 of which were preoperative, and in one case samples were negative preoperatively and positive later.

The authors note a lack of correspondence between culture and PCR results, and consider that the number of organisms involved is low, and that systemic antibiotic prophylaxis for patients who are at risk is a controversial matter. 